\author{
Submitted: \\ 16.03.2021 \\ Accepted: \\ 28.04.2021 \\ Published: \\ 07.06.2021

\section{Ultrasound-guided musculoskeletal interventional procedures around the elbow, hand and wrist excluding carpal tunnel procedures} \\ Silvia Tortora1, Carmelo Messina²,3, Domenico Albano ${ }^{3,4}$, \\ Francesca Serpi ${ }^{1}$, Angelo Corazza ${ }^{3}$, Gianpaolo Carrafiello ${ }^{5,6}$, \\ Luca Maria Sconfienza ${ }^{2,3}$, Salvatore Gitto² \\ ${ }^{1}$ Scuola di Specializzazione in Radiodiagnostica, Università degli Studi di Milano, Italy \\ ${ }^{2}$ Dipartimento di Scienze Biomediche per la Salute, Università degli Studi di Milano, Italy \\ ${ }^{3}$ Unità di Radiologia Diagnostica e Interventistica, IRCCS Istituto Ortopedico Galeazzi, Italy \\ ${ }^{4}$ Sezione di Scienze Radiologiche, Dipartimento di Biomedicina, Neuroscienze e Diagnostica \\ Avanzata, Università degli Studi di Palermo, Italy \\ ${ }^{5}$ Dipartimento di Scienze della Salute, Università degli Studi di Milano, Italy \\ ${ }^{6}$ Unit of Radiology, IRCCS Cà Granda Ospedale Maggiore Policlinico, Italy \\ Correspondence: Salvatore Gitto,e-mail: sal.gitto@gmail.com
}

\section{Keywords}

elbow,

finger,

injection,

ultrasound,

wrist

DOI: $10.15557 / J o U .2021 .0027$

\begin{abstract}
Ultrasound is a fast, reliable and radiation-free method for the assessment of a wide range of pathological conditions, as well as for the guidance of percutaneous interventional procedures around the elbow, hand and wrist. Intraarticular and periarticular interventional procedures can be easily performed under continuous ultrasound monitoring to ensure correct needle positioning and medication delivery to a specific target. The most common ultrasound-guided procedures performed around the elbow, wrist, and hand are described in this review, excluding carpal tunnel procedures. Specifically, elbow steroid injections are performed in patients with inflammatory disorders, while hyaluronic acid can be administered in case of osteoarthritis. Septic olecranon bursitis requires percutaneous drainage for diagnosis and appropriate treatment. Dry needling and injection of regenerative medications, such as blood derivatives, are among the treatment options for lateral epicondylosis. Steroid injections are performed to give symptom relief in patients with ulnar neuropathy at the elbow. Hand and wrist steroid injections are performed in case of osteoarthritis, subsequently followed by hyaluronic acid injections, and in inflammatory disorders. Wrist ganglia can be safely aspirated and injected with steroids under ultrasound guidance. De Quervain's tenosynovitis and trigger finger are chronic tenosynovitides of the wrist and digits, respectively, which can be treated with steroid injections, subsequently followed by hyaluronic acid injections. In conclusion, proper knowledge of the musculoskeletal disorders around the elbow, hand and wrist, as well as US-guided treatment options and operator training, are prerequisites to achieve good outcomes.
\end{abstract}

\section{Introduction}

Musculoskeletal interventional procedures of the upper limb have been performed for several years using a blinded or landmark-guided approach due to the relatively superficial position of the anatomical landmarks. However, recent European Society of Musculoskeletal Radiology (ESSR) guidelines have shown that interventional procedures performed under ultrasound (US) guidance bring better results in terms of accuracy, efficacy, and the risk-benefit ratio(1,2). US is a fast, accessible, reliable, and radiation-free method for the anatomical and dynamic assessment of the softtissue structures of the upper limb ${ }^{(3)}$, which allows for the identification and treatment guidance in a wide range of 
pathological conditions. It is, however, operator-dependent and, as such, the knowledge of the anatomy and pathologies, as well as proper operator training, are necessary preconditions to make the diagnosis correctly and perform the percutaneous treatment accurately. In this article, the US-guided interventional procedures around the elbow, hand and wrist are reviewed, excluding carpal tunnel procedures, and a brief disease description, treatment indications and techniques (including procedural approaches, patient positioning, and needles used in the clinical routine) are discussed.

\section{Elbow}

\section{Intraarticular injection}

The elbow is formed by the humeroulnar, humeroradial and proximal radioulnar joints covered by a single joint capsule $^{(4)}$. The elbow joint can be affected by different pathological conditions, such as rheumatoid arthritis, crystal-induced arthropathies, and osteoarthritis, which present with joint effusion. In such cases, joint injections can be performed for therapeutic or diagnostic purposes. Particularly, steroids with or without a local anesthetic are usually injected in cases involving inflammatory conditions, while hyaluronic acid can be administered in case of osteoarthritis. A local anesthetic can be injected to give pain relief and then confirm the intraarticular origin of pain $^{(4)}$. US clearly depicts joint effusion, intraarticular loose bodies, and synovial hypertrophy, and it can also be used as a guide for injections ${ }^{(4)}$. Cunnington at al. ${ }^{(5)}$ and Kim et al. ${ }^{(6)}$ reported higher accuracy rates for the US-guided $(91 \%$ and $100 \%$, respectively) versus landmark-guided (64\% and $77.5 \%$, respectively) injection approaches.

US-guided elbow joint injections can be performed using an anterior or a posterior approach. Using the posterior approach, the patient lies prone, with the elbow flexed to $90^{\circ}$ and forearm placed over the edge of the examination table. A linear high-frequency transducer is placed short axis to the distal triceps brachii tendon over the posterior olecranon fossa. A 21-gauge needle can be inserted into the joint using an in-plane approach passing beneath the triceps brachii tendon from the lateral to the medial side ${ }^{(4)}$. Using the anterior approach, the patient is seated in front of the operator, with the upper limb placed on the examination table and the elbow flexed to $90^{\circ}$. A linear high-frequency transducer is positioned longitudinal to the radius in order to visualize the humeroradial joint space. Then, a 21-gauge needle is inserted into the joint either using an in-plane approach ${ }^{(7)}$ or vertically with an out-of-plane approach (Fig. 1) ${ }^{(4)}$.

\section{Olecranon bursitis}

The olecranon bursa is located superficial to the olecranon process and, under healthy conditions, it is not sonographically visible. Olecranon bursitis is an inflammatory process caused by direct trauma, infection or non-infectious

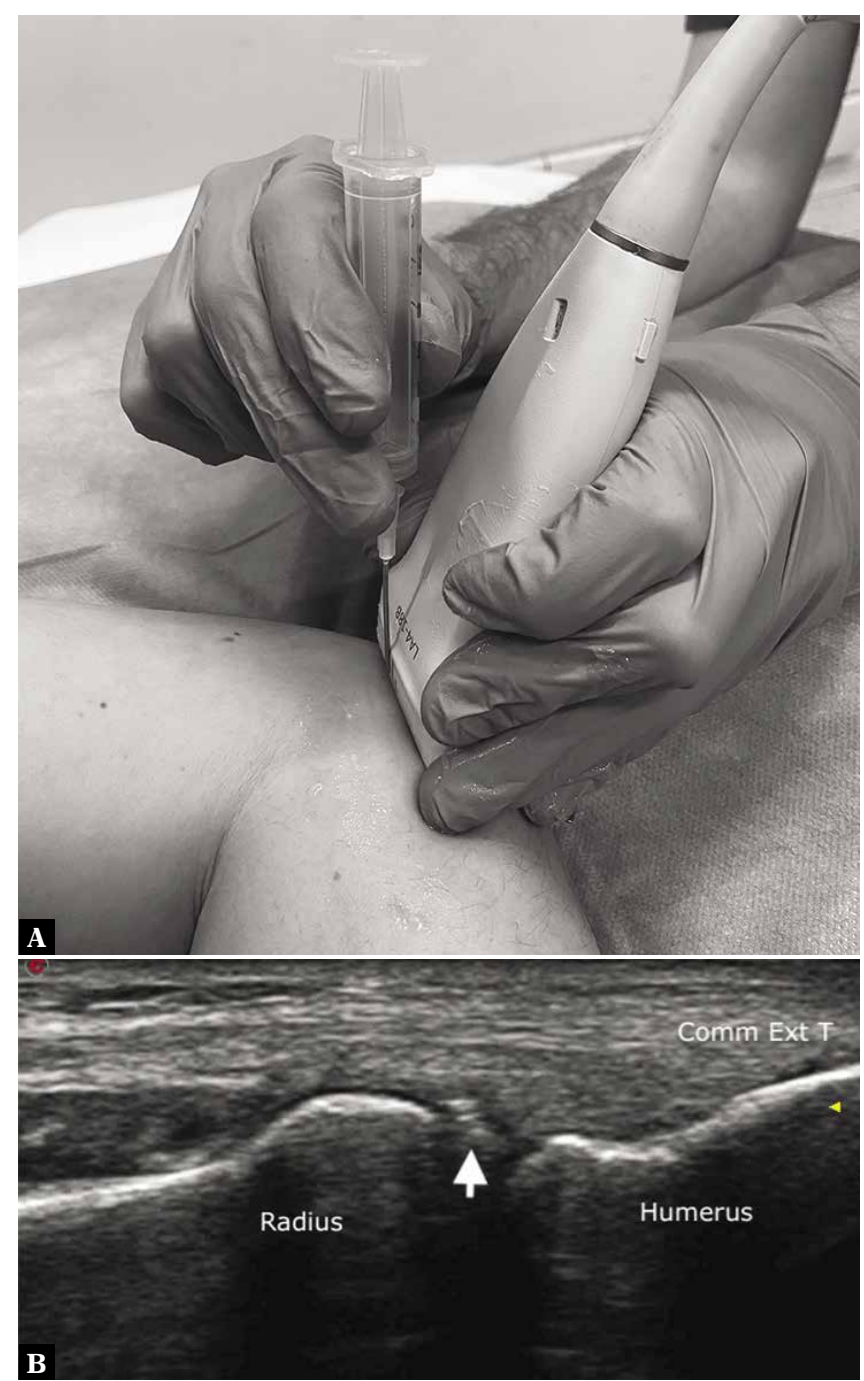

Fig. 1. Elbow joint injection. A. Transducer and needle positioning for simulated elbow joint injection. B. On a longitudinal US scan, the humeroradial joint is depicted underneath the common extensor tendon (Comm Ext T). A 21-gauge needle (arrow) is inserted into the joint using an out-of-plane approach

inflammatory disorders. It is characterized by fluid effusion associated with hypertrophic synovium ${ }^{(7)}$.

There is currently no standard of care for olecranon bursitis. In patients with aseptic bursitis, it is possible to resort to conservative treatment (ice, rest, oral anti-inflammatory medications, and compression dressings), and in non-resolving cases, US-guided drainage with or without a steroid injection can be applied. The use of steroids is, however, controversial. In a review, it was reported that the initiation of steroids for aseptic bursitis was associated with an increase in complications, such as skin atrophy, without an improvement in outcome ${ }^{(8)}$. Additionally, steroid injections may predispose the distal triceps brachii tendon to rupture ${ }^{(7)}$. Septic bursitis requires drainage for the diagnosis and appropriate treatment ${ }^{(7)}$.

US can be used as a guide for both therapeutic and diagnostic percutaneous procedures. The patient lies prone, 

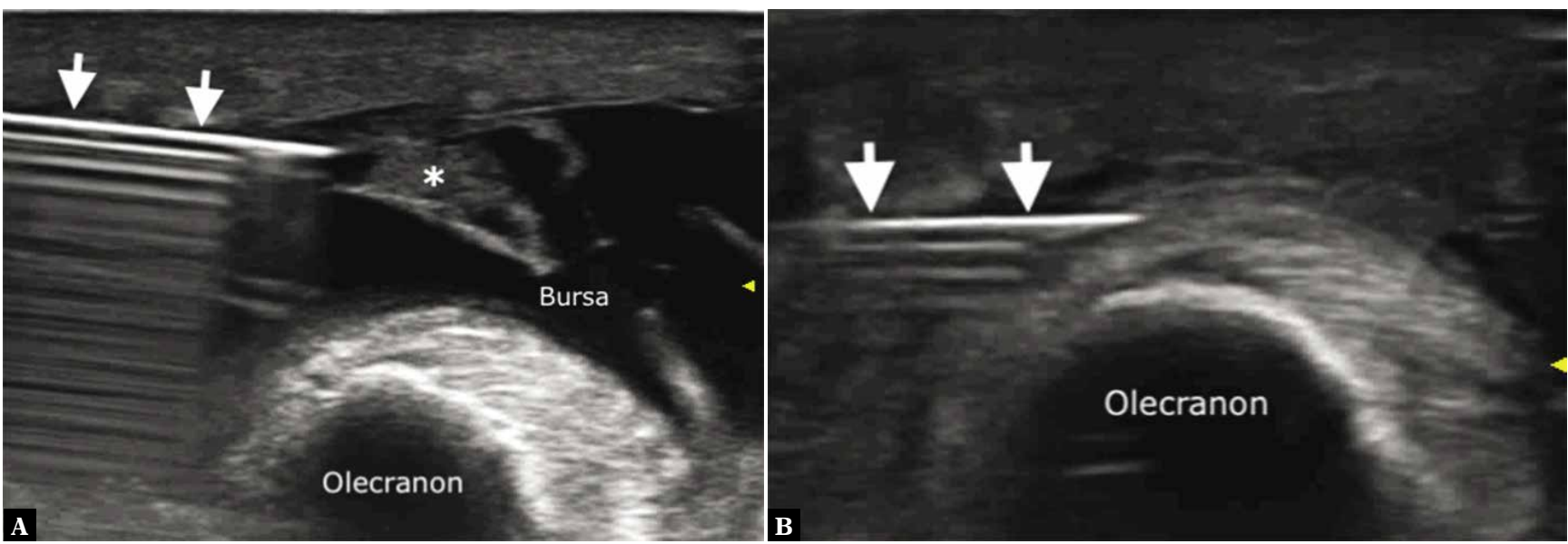

Fig. 2. Olecranon bursitis aspiration. Fluid and synovial hypertrophy (asterisk) are seen within the inflamed olecranon bursa. A. An 18-gauge needle (arrows) is inserted into the bursa using an in-plane approach and $\boldsymbol{b}$ fluid is aspirated for diagnostic purposes

with the arm along the body. The bursitis is visualized using axial and longitudinal US scans with a linear highfrequency probe. Minimum transducer pressure must be used to avoid fluid moving away from the field of view. Then, a 16/18-gauge needle is inserted into the bursa, and the fluid is aspirated (Fig. 2). When applicable, $1 \mathrm{~mL}$ of a low-solubility steroid can be injected into the bursa. A compression bandage is then applied ${ }^{(4)}$.

\section{Lateral epicondylosis}

Lateral epicondylosis is the most common cause of lateral elbow pain, affecting between 1 and 3\% of adults ${ }^{(9)}$. The term "epicondylosis" is more appropriate than "epicondylitis", as the process is mainly degenerative rather than inflammatory. The pathology affects the common tendon attachment of the four extensor muscles of the forearm (extensor carpi radialis brevis, extensor digitorum communis, extensor digiti minimi, and extensor carpi ulnaris) to the lateral epicondyle. The extensor carpi radialis brevis tendon is the most commonly affected ${ }^{(9)}$. Lateral epicondylosis includes the following stages: stage 1, when a transient inflammatory response occurs; stage 2 , characterized by fibroblastic hyperplasia, hyperplasia of blood vessels and breakdown of collagen fibers; stage 3 , when partial or full-thickness tendon tears occur; and stage 4 , characterized by fibrosis and calcification ${ }^{(10)}$

The first-line treatment is conservative, including rest, oral anti-inflammatory medications, and physiotherapy ${ }^{(11)}$. Steroid injections have also been performed in clinical practice, either blindly or under US guidance. In a randomized clinical trial, Bisset et al. found that steroid injections had no significant long-term benefit compared to conservative treatment, and were associated with much higher rates of recurrence than physiotherapy (72\% vs. $8 \%$, respectively) ${ }^{(11)}$. Currently, the use of steroid injections is not advised ${ }^{(1)}$. As reported in the ESSR guidelines, steroids can be injected in selected cases, for example in individuals seeking immediate pain relief or those unable to undergo physiotherapy. However, patients should be advised of the potential long-term harmful effects, such as tendon damage and skin atrophy ${ }^{(1)}$. The second-line treatment includes dry needling (repeated tendon punctures in the degenerated areas), platelet-rich plasma injection, autologous blood injection, prolotherapy (injection of irritative agents into the degenerated tendon areas) and sclerotherapy (injection of sclerozing agents causing thrombosis and vessel occlusion $)^{(1,4)}$. In a randomized clinical trial by Mishra et al. comparing dry needling with and without platelet-rich plasma, no differences in pain scores were noted at 12 weeks. At 24 weeks, however, improvements were observed in patients treated with platelet-rich plasma compared to the control group $^{(12)}$.

The US-guided procedure is performed similarly regardless of the injected agent. The patient is positioned in the seated or supine position, with the elbow flexed to approximately $90^{\circ}$. A linear high-frequency transducer is positioned long axis to the common extensor tendon. For prolotherapy and sclerotherapy, local anesthesia is performed prior to the injection. A 21-gauge needle is inserted distal-to-proximal into the degenerated area of the tendon using an in-plane approach. Then, a selected medication is injected or, in the case of dry needling, a total of 15-20 punctures are performed (Fig. 3)(4).

\section{Ulnar neuropathy}

Ulnar nerve neuropathy at the elbow is the second most common entrapment neuropathy of the upper extremity after carpal tunnel syndrome, with an incidence of 24.7 cases per 100,000 per year ${ }^{(13)}$. The nerve is compressed at the level of the cubital tunnel, a fibro-osseous tunnel formed by the olecranon process laterally, medial epicondyle medially, elbow joint capsule anteriorly, and Osborne's retinaculum posteriorly. The shape of the cubital tunnel changes with elbow flexion and external factors, resulting in chronic pressure and consequent tunnel narrowing ${ }^{(14)}$. The typical symptoms are pain, weakness and paresthesia in the fourth and fifth fingers, as well as numbness of the dorsal ulnar side of the hand ${ }^{(14)}$. 


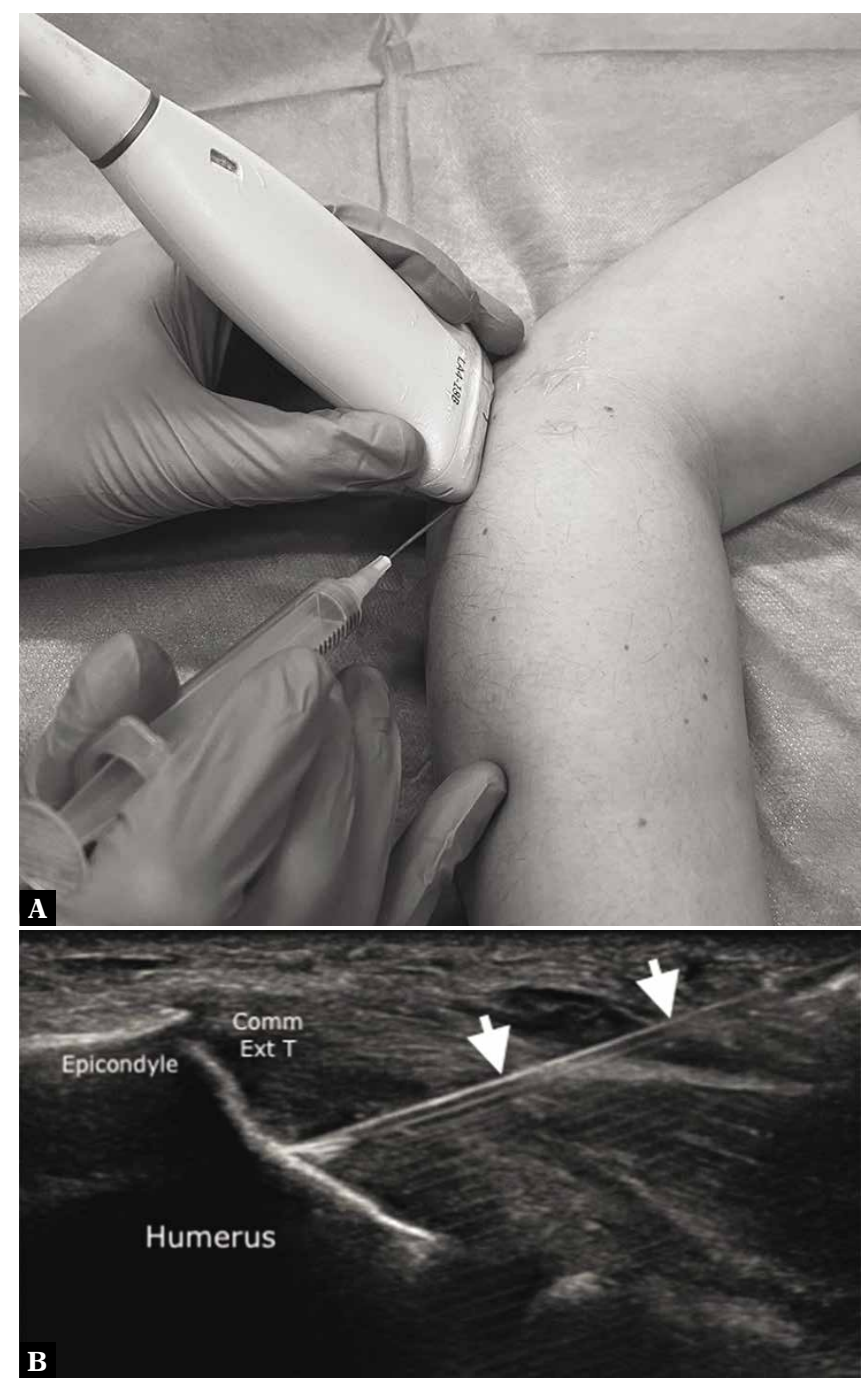

Fig. 3. Common extensor tendon dry needling. A. Transducer and needle positioning for simulated common extensor tendon dry needling. B. The common extensor tendon (Comm Ext T) is thickened and degenerated at its attachment to the lateral epicondyle. A 21-gauge needle (arrows) is inserted into the degenerated areas of the tendon using an in-plane distal-to-proximal approach, and repeated punctures are performed

The first-line treatment for patients with mild to moderate symptoms is conservative, and consists of patient education on how to avoid provocative activities and elbow positions, splinting and local steroid injections ${ }^{(15)}$. Steroid injections may improve symptoms ${ }^{(16)}$, even though some studies reported no significant differences in terms of clinical outcomes compared to the placebo ${ }^{(17)}$. Surgery is required in patients with severe symptoms or non-responsive to conservative treatment ${ }^{(15)}$.

Steroid injections can be performed under US guidance. The US-guided procedure is performed with the patient supine, with the arm above the head, and the elbow flexed. Using a linear high-frequency transducer, the ulnar nerve is visualized in the transverse plane at the cubital tunnel level. The thickest part of the nerve is identified, and a steroid is injected around it $^{(16)}$.

\section{Wrist}

\section{Radiocarpal joint injection}

The radiocarpal joint is a synovial joint between the radius and the proximal row of the carpal bones. It is stabilized by several ligaments and muscle-tendon units, as well as by the triangular fibrocartilage complex ${ }^{(18)}$. US guidance can be used for therapeutic injections in patients with joint disorders, such as osteoarthritis and inflammatory arthritis, as well as for diagnostic purposes, if joint fluid aspiration is needed. The accuracy rates of landmark-guided and US-guided injection into the radiocarpal joint range from $25 \%$ to $97 \%$, and $79 \%$ to $94 \%$, respectively ${ }^{(19)}$. In case of both inflammatory and degenerative arthropathies, an intraarticular steroid injection is beneficial. In degenerative conditions, it can be followed by an intraarticular injection of hyaluronic acid approximately after 2 weeks ${ }^{(4)}$.

During the US-guided procedure, the patient is placed supine, with the hand pronated and the wrist slightly flexed. Alternatively, the patient is seated in front of the operator, and the hand is placed on the examination table with the palm facing down. A rolled towel can be positioned beneath the wrist to facilitate dorsal joint recess opening. Radiocarpal injections are most frequently performed into the radioscaphoid joint space due to the ease of access and the lack of overlying neurovascular structures. A linear high-frequency transducer is positioned dorsally in the axial plane in order to visualize the Lister's tubercle in the center of the sonogram, and then a rotation of approximately $90^{\circ}$ is made to depict the radiocarpal joint in the long axis. A 25/27-gauge needle is advanced distalto-proximal using an in-plane approach or vertically using an out-of-plane approach (Fig. 4) between the second and third dorsal compartments of the extensor tendons ${ }^{(4,19)}$.

\section{Scaphotrapeziotrapezoidal joint and trapeziometacarpal joint injections}

Scaphotrapeziotrapezoidal and trapeziometacarpal joints are common sites of wrist and hand osteoarthritis ${ }^{(19)}$. Under pathological conditions, US shows joint effusion, synovitis, osteophytes and articular space narrowing. Frequently, flexor carpi radialis tendinopathy is associated with scaphotrapeziotrapezoidal joint osteoarthritis ${ }^{(19)}$. The first-line treatment is conservative (rest, splinting, oral anti-inflammatory medications). Joint injections can be performed for diagnostic or therapeutic purposes under US guidance. The scaphotrapeziotrapezoidal and trapeziometacarpal joint spaces are relatively small. Thus, in case of therapeutic injections, $0.5 \mathrm{~mL}$ of a steroid is enough to provide pain relief, and can be followed by a hyaluronic acid injection after 2 weeks ${ }^{(4)}$.

With regard to the US-guided scaphotrapeziotrapezoidal joint procedure, the patient is seated in front of the operator, with the forearm supinated and the wrist placed over a rolled towel to facilitate wrist-thumb extension. A linear 


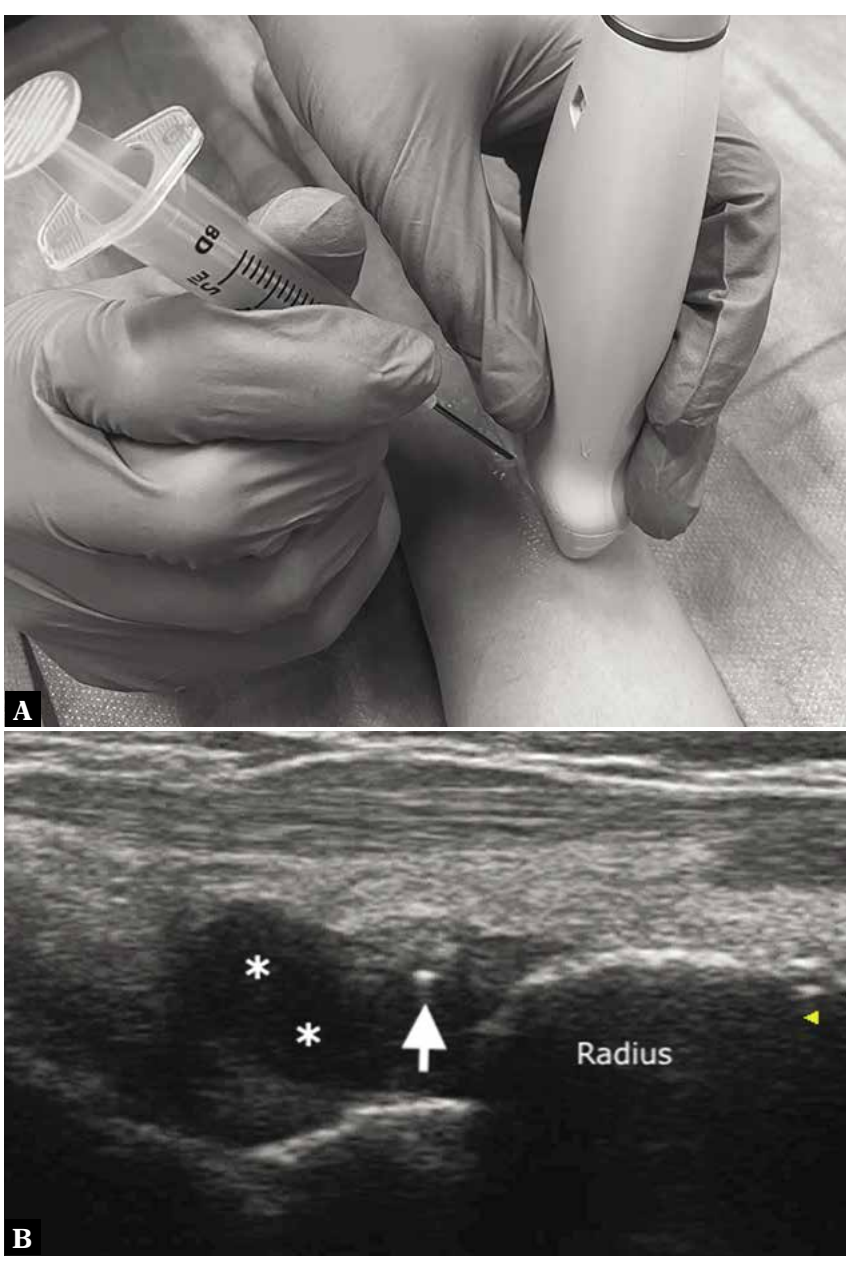

Fig. 4. Radiocarpal joint injection. A. Transducer and needle positioning for simulated radiocarpal joint injection. B. On a longitudinal scan, the radiocarpal dorsal recess is thickened (asterisks) in a rheumatoid arthritis patient. A 25-gauge needle (arrow) is inserted into the joint using an out-of-plane approach to perform joint injection

high-frequency transducer is placed in the longitudinal plane to visualize the scaphotrapeziotrapezoidal joint ${ }^{(19)}$. A 25/27-gauge needle is inserted vertically using an out-ofplane approach $^{(4)}$.

With regard to US-guided trapeziometacarpal joint procedure, the patient is seated in front of the operator with the forearm and wrist in a neutral position between pronation and supination. A linear high-frequency transducer is placed long axis to the first metacarpal and translated proximally over the trapeziometacarpal joint ${ }^{(19)}$. A 25/27gauge needle is inserted vertically into the joint using an out-of-plane approach ${ }^{(4)}$.

\section{Ganglia}

The most common soft-tissue masses of the wrist are benign ganglion cysts ${ }^{(20)}$. They are more frequently located on the dorsal side than the volar side of the wrist (approximately $70 \%$ and $30 \%$ of cases, respectively ${ }^{(20)}$. The etiology of ganglia remains an enigma, although it is possible that their appearance is related to minor trauma leading to mucinous degeneration of the periarticular soft tissues. Ganglion cysts are lined with a connective tissue capsule and contain a viscous gelatin-like fluid ${ }^{(21)}$. From a clinical point of view, ganglia appear as palpable masses that can grow progressively, causing local discomfort and pain ${ }^{(21)}$. The US appearance is predominantly anechoic, oval, round or lobulated, with thin septa and a pedicle that connects the ganglion cyst with the joint capsule ${ }^{(20)}$. Treatment can be percutaneous (aspiration and/or injection), performed blindly or under US guidance, or surgical using an open or arthroscopic approach. Recurrence rates are 33-81\% after landmark-guided percutaneous treatment, 14-19\% after US-guided percutaneous treatment, and $7 \%$ after surgery ${ }^{(1)}$.

US can be used as a guide for percutaneous treatment, although the procedure is painful ${ }^{(1)}$. The patient is seated in front of the operator, with the hand and wrist on the examination table, pronated or supinated, depending on the ganglion cyst location. A linear high-frequency transducer is placed over the ganglion cyst. Doppler imaging allows identifying and avoiding vessels that are close to the ganglion. A thin needle (23-gauge) and a small amount of an anesthetic are used to perform local anesthesia. Then, a 16/18-gauge needle is used to penetrate the ganglion cyst. Lavage with an anesthetic may be necessary to decrease content viscosity and facilitate the aspiration. The viscous fluid is then aspirated. A small amount $(0.5 \mathrm{~mL})$ of a lowsolubility steroid can be injected into the ganglion after the aspiration to facilitate closure of the cyst and relieve pain $^{(4,19)}$. At the end of the procedure, compression taping is applied for 5 to 10 days to minimize steroid reflux and the probability of recurrence ${ }^{(4)}$.

\section{De Quervain's tenosynovitis}

The first dorsal compartment of the wrist contains the abductor pollicis longus and extensor pollicis brevis tendons $^{(18)}$. De Quervain's tenosynovitis is a chronic stenosing tenosynovitis of the first dorsal compartment ${ }^{(22)}$. It is secondary to repetitive microtraumas causing a fibroblastic response with subsequent swelling and thickening of the extensor retinaculum forming the roof the first compartment ${ }^{(22)}$. De Quervain's tenosynovitis presents as a gradual onset of pain along the radial styloid, which is exacerbated by wrist and thumb movements, grasping and raising objects. Ulnar deviation of the wrist with the thumb grasped, known as the Finkelstein's test, is typically pain$\mathrm{ful}^{(22)}$. The diagnosis is based on clinical findings, and US can confirm the diagnosis and depict disease severity. The US findings include thickening of the extensor retinaculum, tendon thickening, synovial sheath fluid effusion or thickening and peritendinous hyperemia on Doppler imaging ${ }^{(23)}$. US also allows identifying an intracompartmental septum, which is an anatomical variation resulting in two sub-compartments. In this case, pathological changes can be confined to only one tendon of the first dorsal compartment, thus requiring the treatment to be targeted accordingly ${ }^{(24)}$. The first-choice treatment is conservative, 

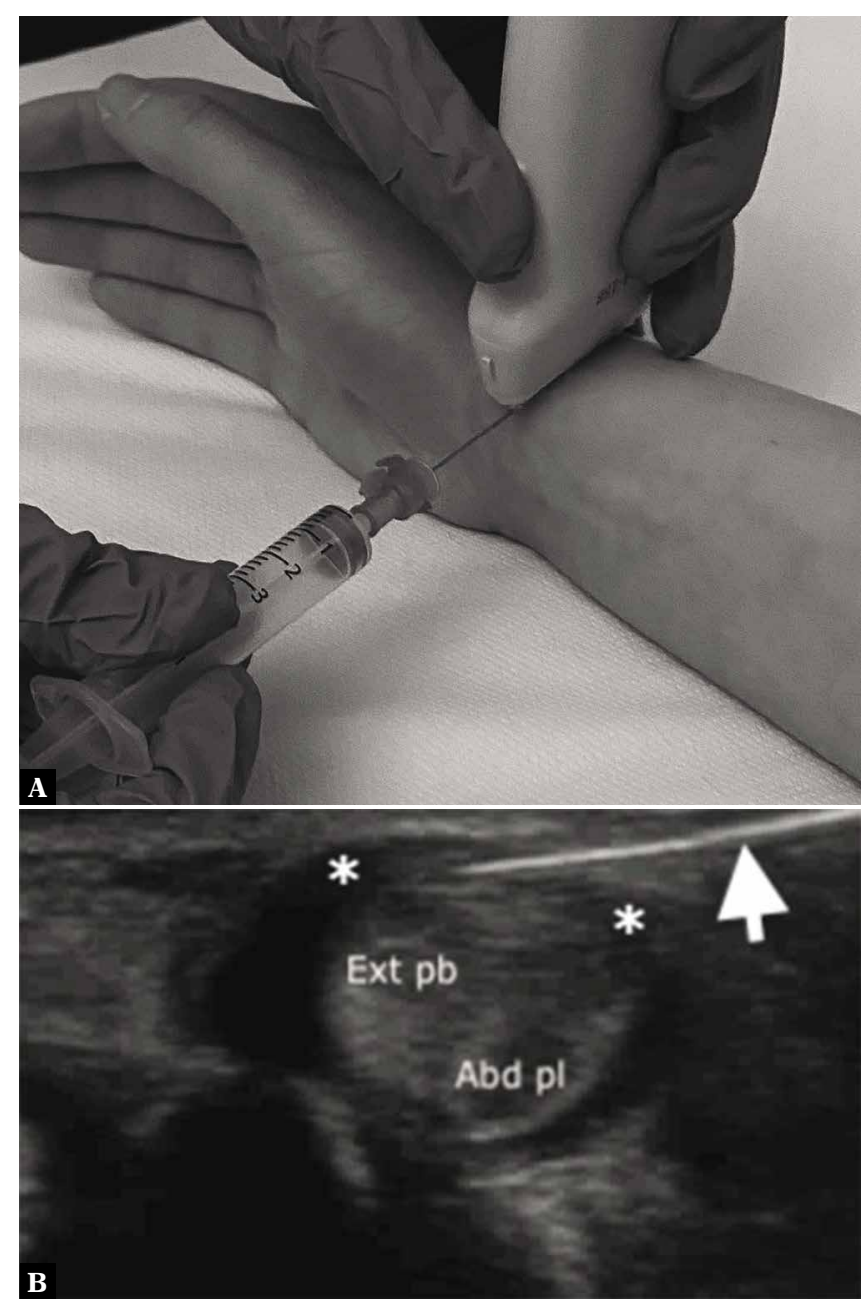

Fig. 5. De Quervain's tenosynovitis injection. A. Transducer and needle positioning for simulated De Quervain's tenosynovitis injection. B. On an axial US scan, the abductor pollicis longus $(\mathrm{Abd} \mathrm{pl})$ and extensor pollicis brevis (Ext pb) tendons are thickened and rounded due to tendinosis. The extensor retinaculum forming the roof of the I extensor compartment (asterisks) is thickened. A 25-gauge needle (arrow) is inserted underneath the retinaculum using an in-plane lateral-to-medial approach

consisting of rest, anti-inflammatory medications and bracing $^{(4)}$. US-guided injection of steroids is a safe and effective method for alleviating pain, with symptom relief reported in $94-97 \%$ and $94-96 \%$ of patients at an average followup of 6 weeks and 6 months, respectively ${ }^{(1)}$. Orlandi et al. showed that a steroid injection followed by a hyaluronic acid injection 2 weeks apart improves the clinical outcome compared to a steroid injection alone ${ }^{(25)}$.

During the US-guided procedure, the patient is seated in front of the operator, with the wrist placed on the examination table in a neutral position between pronation and supination. Using a linear high-frequency transducer, the first extensor compartment is visualized in the axial plane on the dorsal-lateral aspect of the radius. A 23/25-gauge needle is inserted underneath the thickened retinaculum using an in-plane lateral-to-medial approach (Fig. 5) ${ }^{(26)}$. Lapegue et al. recently performed a prospective study on
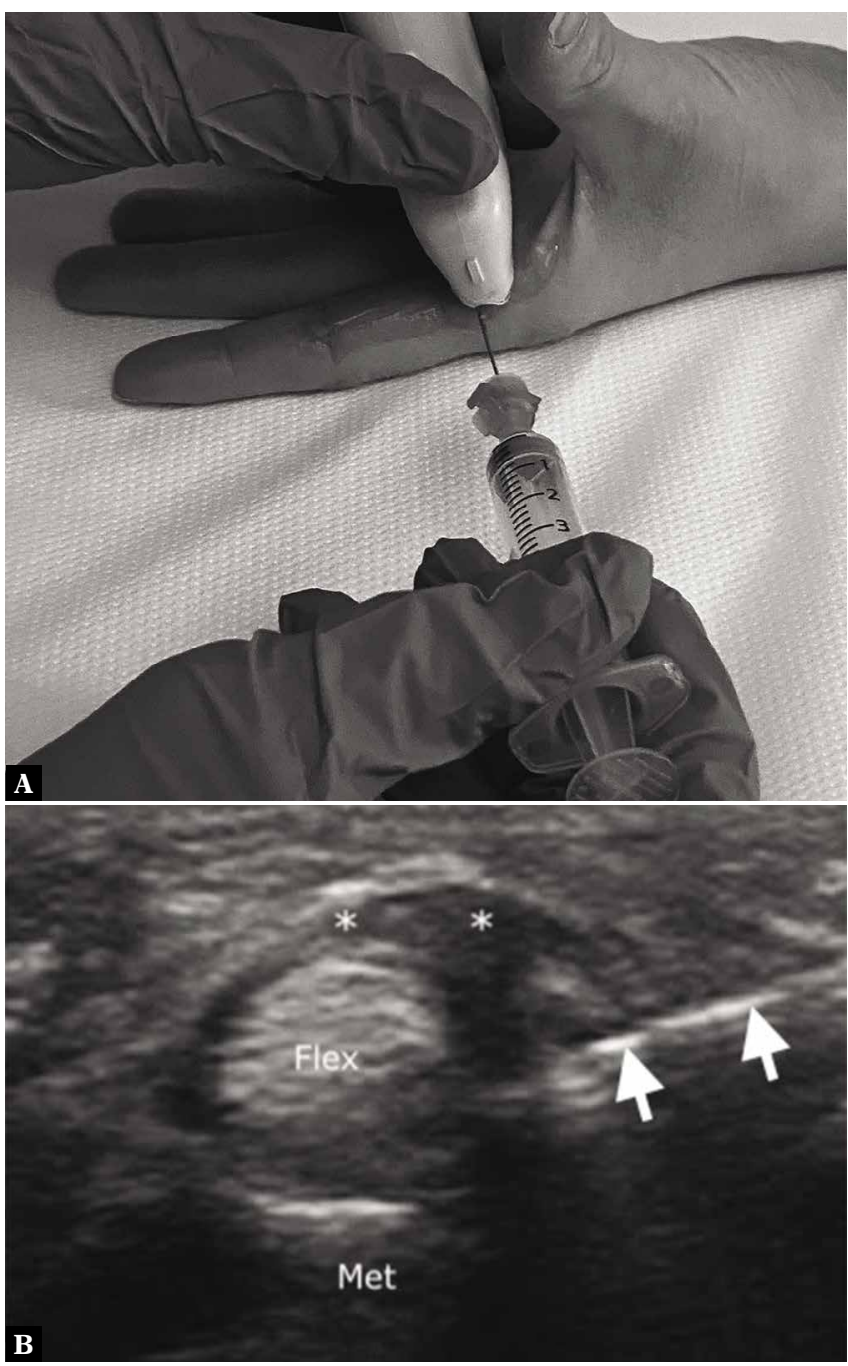

Fig. 6. Trigger finger injection. A. Transducer and needle positioning for simulated trigger finger injection. B. At the level of the metacarpal head (Met), the flexor tendons (Flex) are shown, and the A1 pulley (asterisks) is thickened. A 25-gauge needle (arrows) is inserted underneath the pulley using an in-plane axial approach

35 patients, and proposed US-guided percutaneous release of the first compartment as an alternative treatment to surgery. A 21-gauge needle was used to perform retinaculum release under US guidance ${ }^{(27)}$.

\section{Hand}

\section{Metacarpophalangeal and interphalangeal joint injections}

The metacarpophalangeal and interphalangeal joints can be affected by osteoarthritis, both primary and secondary to traumas, as well as inflammatory disorders. These conditions cause pain and impaired function during daily activities $^{(19)}$. In case of conservative treatment failure, steroid injections can be performed under US guidance ${ }^{(19)}$. During 
the US-guided procedure, the patient is seated in front of the operator, with the forearm and wrist pronated on the examination table, and a rolled towel is placed underneath the hand to facilitate finger flexion. A linear high-frequency transducer is positioned long axis to the metacarpal or phalanx, with the dorsal joint recess visualized in the center of the sonogram. A 25/27-gauge needle is inserted using an out-of-plane approach ${ }^{(19)}$. As the joint space is small, 0.5 $\mathrm{mL}$ of a steroid is sufficient to provide symptom relief ${ }^{(26)}$.

\section{Trigger finger}

Trigger finger is chronic stenosing tenosynovitis of the flexor tendons of the digits, caused by repeated microtraumas resulting in A1 pulley thickening ${ }^{(23)}$. The clinical presentation includes painful blockage and triggering of the digit from flexion to extension ${ }^{(23)}$. The main US findings are A1 pulley thickening and flexor tendon thickening with loss of fibrillar echotexture ${ }^{(28)}$. The first-line treatment includes rest, anti-inflammatory medications, and splinting. Then, US-guided steroid injections can be performed. Surgical release is the standard of care for the management of severe cases $^{(26)}$.

During the US-guided procedure, the patient is seated in front of the operator, with the hand supinated on the examination table. A linear high-frequency transducer is positioned long axis to the flexor tendons at the level of the metacarpophalangeal joint in order to visualize the thickened A1 pulley. A 25/27-gauge needle is inserted into the tendon synovial sheath using an in-plane approach, either longitudinal distal-to-proximal or axial (Fig. 6). A small amount of an anesthetic is injected first to confirm needle placement, followed by $1 \mathrm{~mL}$ of a steroid ${ }^{(26)}$. An US-guided technique combining a steroid injection and a 10-day-delayed injection of hyaluronic acid has been described to provide pain relief and improve the range of motion $^{(29)}$. Lapegue et al. proposed US-guided percutaneous release of the A1 pulley as an alternative option to surgery. A 21-gauge needle was used to perform pulley release under US guidance ${ }^{(30)}$.

\section{Conclusions}

Musculoskeletal interventional procedures around the elbow, hand and wrist can be performed under US guidance to treat different disorders. Proper knowledge of the pathologies and US-guided techniques, as well as operator training, are prerequisites to achieve good outcomes.

\section{Conflict of interest}

The authors do not report any financial or personal connections with other persons or organizations, which might negatively affect the contents of this publication and/or claim authorship rights to this publication.

\section{References}

1. Sconfienza LM, Adriaensen M, Albano D, Aparisi Gómez MP, Bazzocchi A, Beggs I et al.: Clinical indications for image-guided interventional procedures in the musculoskeletal system: a Delphi-based consensus paper from the European Society of Musculoskeletal Radiology (ESSR) - part II, elbow and wrist. Eur Radiol 2020; 30: 2220-2230.

2. Sconfienza LM, Adriaensen M, Albano D, Allen G, Aparisi Gómez MP, Bazzocchi A et al.: Clinical indications for image guided interventional procedures in the musculoskeletal system: a Delphi-based consensus paper from the European Society of Musculoskeletal Radiology (ESSR) - part III, nerves of the upper limb. Eur Radiol 2020; 30: 1498-1506.

3. Gitto S, Messina C, Mauri G, Aliprandi A, Sardanelli F, Sconfienza LM: Dynamic high-resolution ultrasound of intrinsic and extrinsic ligaments of the wrist: how to make it simple. Eur J Radiol 2017; 87: 20-35.

4. Sconfienza LM, Chianca V, Messina C, Albano D, Pozzi G, Bazzocchi A: Upper limb interventions. Radiol Clin North Am 2019; 57: 1073-1082.

5. Cunnington J, Marshall N, Hide G, Bracewell C, Isaacs J, Platt P et al.: A randomised, controlled, double blinded study of ultrasound guided corticosteroid joint injection in patients with inflammatory arthritis. Arthritis Rheum 2010; 62: 1862-1869.

6. Kim TK, Lee JH, Park KD, Lee SC, Ahn J, Park Y: Ultrasound versus palpation guidance for intra-articular injections in patients with degenerative osteoarthritis of the elbow. J Clin Ultrasound 2013; 41: 479-485.

7. Sussman WI, Williams CJ, Mautner K: Ultrasound-guided elbow procedures. Phys Med Rehabil Clin N Am 2016; 27: 573-587.

8. Sayegh ET, Strauch RJ: Treatment of olecranon bursitis: a systematic review. Arch Orthop Trauma Surg 2014; 134: 1517-1536.

9. Faro F, Wolf JM: Lateral epicondylitis: review and current concepts. J Hand Surg Am 2007; 32: 1271-1279.

10. Lenoir H, Mares O, Carlier Y: Management of lateral epicondylitis. Orthop Traumatol Surg Res 2019; 105: S241-S246.

11. Bisset L, Beller E, Jull G, Brooks P, Darnell R, Vicenzino B: Mobilisation with movement and exercise, corticosteroid injection, or wait and see for tennis elbow: randomised trial. BMJ 2006; 333: 939.

12. Mishra AK, Skrepnik NV, Edwards SG, Jones GL, Sampson S, Vermillion DA et al.: Efficacy of platelet-rich plasma for chronic tennis elbow: a double-blind, prospective, multicenter, randomized controlled trial of 230 patients. Am J Sports Med 2014; 42: 463-471.

13. Mondelli M, Giannini F, Ballerini M, Ginanneschi F, Martorelli E: Incidence of ulnar neuropathy at the elbow in the province of Siena (Italy). J Neurol Sci 2005; 234: 5-10.

14. Klauser A, Buzzegoli T, Taljanovic M, Strobl S, Rauch S, Teh J et al.: Nerve entrapment syndromes at the wrist and elbow by sonography. Semin Musculoskelet Radiol 2018; 22: 344-353.

15. Nakashian MN, Ireland D, Kane PM: Cubital tunnel syndrome: current concepts. Curr Rev Musculoskelet Med 2020; 13: 520-524.

16. Alblas CL, van Kasteel V, Jellema K: Injection with corticosteroids (ultrasound guided) in patients with an ulnar neuropathy at the elbow, feasibility study. Eur J Neurol 2012; 19: 1582-1584.

17. vanVeen KEB, Alblas KCL, Alons IME, Kerklaan JP, Siegersma MC, Wesstein $\mathrm{M}$ et al.: Corticosteroid injection in patients with ulnar neuropathy at the elbow: a randomized, double-blind, placebo-controlled trial. Muscle Nerve 2015; 52: 380-385.

18. Gitto S, Draghi F: Normal sonographic anatomy of the wrist with emphasis on assessment of tendons, nerves, and ligaments. J Ultrasound Med 2016; 35: 1081-1094.

19. Colio SW, Smith J, Pourcho AM: Ultrasound-guided interventional procedures of the wrist and hand. Phys Med Rehabil Clin N Am 2016; 27 : 589-605.

20. Gitto S, Lee SC, Miller TT: Ultrasound-guided percutaneous treatment of volar radiocarpal ganglion cysts: Safety and efficacy. J Clin Ultrasound 2019; 47: 339-344. 
21. Gude W, Morelli V: Ganglion cysts of the wrist: pathophysiology, clinical picture, and management. Curr Rev Musculoskelet Med 2008; 1: 205-211.

22. Ilyas AM, Ast M, Schaffer AA, Thoder J: De Quervain tenosynovitis of the wrist. J Am Acad Orthop Surg 2007; 15: 757-764.

23. Gitto S, Draghi AG, Draghi F: Sonography of non-neoplastic disorders of the hand and wrist tendons. J Ultrasound Med 2018; 37: 51-68.

24. Choi S-J, Ahn JH, Lee Y-J, Ryu DS, Lee JH, Jung SM et al.: De Quervain disease: US identification of anatomic variations in the first extensor compartment with an emphasis on subcompartmentalization. Radiology 2011; 260: 480-486.

25. Orlandi D, Corazza A, Fabbro E, Ferrero G, Sabino G, Serafini G et al.: Ultrasound-guided percutaneous injection to treat de Quervain's disease using three different techniques: a randomized controlled trial. Eur Radiol 2015; 25: 1512-1519.
26. Orlandi D, Corazza A, Silvestri E, Serafini G, Savarino EV, Garlaschi G et al.: Ultrasound-guided procedures around the wrist and hand: how to do. Eur J Radiol 2014; 83: 1231-1238.

27. Lapègue $\mathrm{F}$, André $\mathrm{A}$, Pasquier Bernachot $\mathrm{E}$, Akakpo EJ, Laumonerie $\mathrm{P}$, Chiavassa-Gandois $\mathrm{H}$ et al.: US-guided percutaneous release of the first extensor tendon compartment using a 21-gauge needle in de Quervain's disease: a prospective study of 35 cases. Eur Radiol 2018; 28: 3977-3985.

28. Bianchi S, Gitto S, Draghi F: Ultrasound features of trigger finger: review of the literature. J Ultrasound Med 2019; 38: 3141-3154.

29. Callegari L, Spanò E, Bini A, Valli F, Genovese E, Fugazzola C: Ultrasound-guided injection of a corticosteroid and hyaluronic acid. Drugs R D 2011; 11: 137-145.

30. Lapègue $\mathrm{F}$, André $\mathrm{A}$, Meyrignac $\mathrm{O}$, Pasquier-Bernachot $\mathrm{E}$, Dupré $\mathrm{P}$, Brun $\mathrm{C}$ et al.: US-guided percutaneous release of the trigger finger by using a 21-gauge needle: a prospective study of 60 cases. Radiology 2016; 280: 493-499. 